
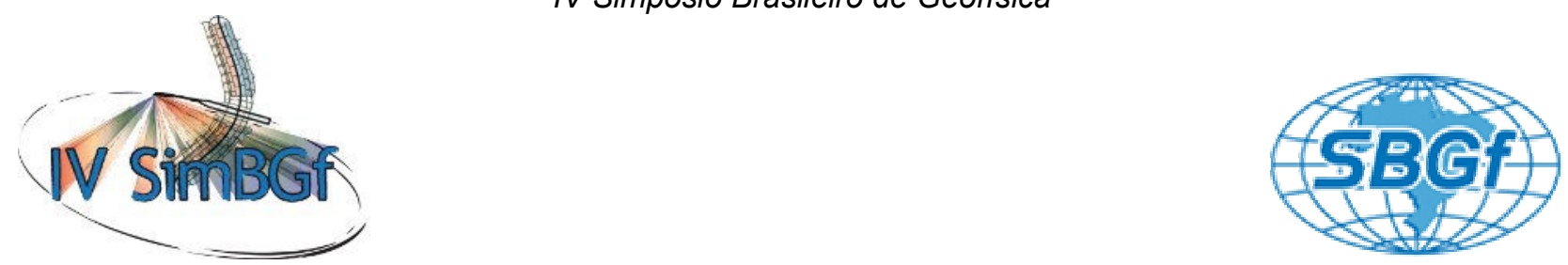

\title{
Uma abordagem sobre aplicação de métodos geoestatísticos para processamento de dados geoquímicos como apoio à seleção de anomalias geofísicas: exemplo na Província Mineral do Tapajós
}

Thais Andressa Carrino, LGA-UnB; Sérgio Sacani Sancevero, ROXAR; Adalene Moreira Silva, LGA-UnB; Nilson Francisquini Botelho, IG-UnB; Marcelo Pinto, Mapex - Mineração, Importação \& Exportação Ltda.; Luiz Carlos Pinheiro Clerot, Mapex - Mineração, Importação \& Exportação Ltda.

Copyright 2010, SBGf - Sociedade Brasileira de Geofísica

Este texto foi preparado para a apresentação no IV Simpósio Brasileiro de Geofísica, Brasilia, 14 a 17 de novembro de 2010. Seu conteúdo foi revisado pelo Comitê Técnico do IV SimBGf, mas não necessariamente representa a opinião da SBGf ou de seus associados. É proibida a reprodução total ou parcial deste material para propósitos comerciais sem prévia autorização da $S B G$.

\section{Resumo}

Esse artigo aborda o uso de três técnicas geoestatísticas a para geração de mapas de teor de ouro em solo no Prospecto 12 de Outubro, localizado na Província Mineral do Tapajós. As técnicas abordadas consistem na krigagem ordinária, krigagem indicatriz e simulação indicatriz seqüencial. Como resultado, observou-se que o uso de técnicas de simulação estocástica permite a manutenção dos dados amostrais originais, ao contrário de métodos suavizadores, como krigagens, compreendendo uma poderosa ferramenta para a prospecção geoquímica que deve ser, recomendavelmente, associada a outros tipos de dados, como os geofísicos nas etapas iniciais de prospecção.

\section{Introdução}

A aquisição de dados geoquímicos equivale a uma das etapas iniciais de follow-up em um projeto de mineração. Estes dados devem ser processados de maneira adequada para prover informações seguras e que mantenham as informações originais. Métodos geoestatísticos, tais como as técnicas de estimativas pontuais e as estocásticas, possuem um papel relevante para aplicações em dados geoquímicos, a fim de integração com informações geofísicas para refino interpretativo do potencial mineral de uma região.

Nesse sentido, este artigo enfoca a comparação de diferentes técnicas geoestatísticas aplicadas em dados geoquímicos de ouro em solo amostrados na região do Prospecto 12 de Outubro, localizado na porção noroeste da Província Mineral do Tapajós (Figura 1). A região do prospecto 12 de Outubro, regionalmente, é afetada por estruturas de direção NE-SW, além de estar inclusa no domínio de rochas vulcânicas ácidas paleoproterozóicas do Grupo Iriri e de vulcânicas intermediárias da Formação Bom Jardim (Klein et al., 2001). As áreas mineralizadas da região do Prospecto 12 de Outubro são caracterizadas por intensa silicificação das rochas vulcânicas ácidas, como riolitos e riodacitos. Neste contexto, foram identificadas em campo brechas hidrotermais e zonas de stockworks, marcadas por vênulas de quartzo centimétricas, nas quais é possível observar a sulfetação com predomínio de pirita, estas com tamanho em torno de 1 a $2 \mathrm{~mm}$. A cava principal é denominada de Main Breccia Pit e explorada pela empresa Mapex Mineração, Importação e Exportação Ltda., possuindo direção NE-SW, a mesma direção preferencial dos stockworks, em torno de N66E.

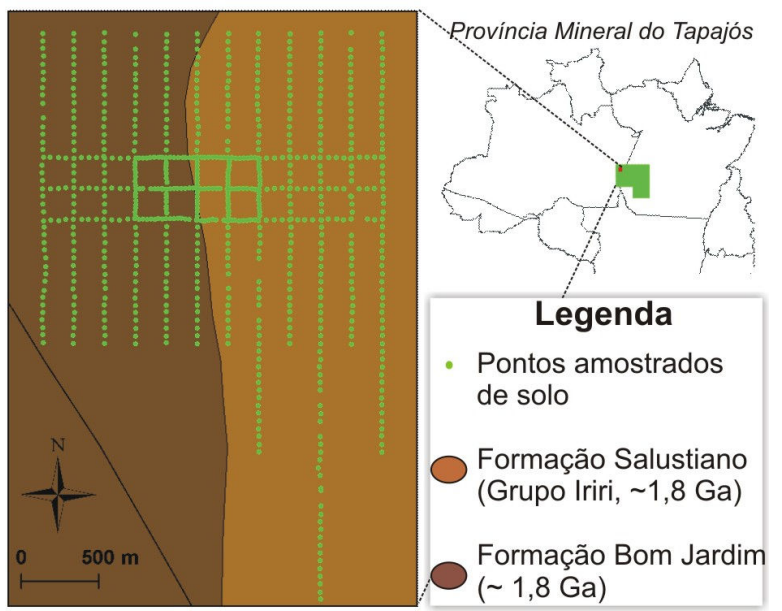

Figura 1. Localização da área de estudo na Província Mineral do Tapajós (prospecto 12 de Outubro), e disposição da malha de solos.

\section{Processamento de dados geoquímicos}

Os dados geoquímicos para ouro em solo foram processados por meio da krigagem ordinária, da krigagem indicatriz e da simulação indicatriz sequencial.

A malha geoquímica consta de 680 amostras coletadas seguindo-se uma amostragem $\mathrm{N}$-S aproximadamente a cada $50 \mathrm{~m}$, sendo na parte central reduzida a cerca de 25 m. A amostragem E-W foi realizada a cada $200 \mathrm{~m}$ em geral, e na porção central, variável entre $50 \mathrm{~m}$ e $25 \mathrm{~m}$ (Figura 1). Para a geração dos grids, optou-se, portanto, pelo emprego de células de $25 \mathrm{~m}$.

\section{Avaliação geoestatística e aplicação de krigagens}

A primeira etapa da avaliação geoestatística aplicada às variáveis contínuas de ouro foi referente à análise variográfica, que compreende uma técnica de análise estrutural e espacial das variáveis regionalizadas 
(Matheron, 1963). O variograma mede a variabilidade a partir das distâncias, utilizando-se do passo (h), um vetor que contabiliza a magnitude da separação dos dados. Matematicamente, representa uma variância calculada para várias distâncias, sendo que o comportamento desta variância determina o padrão de continuidade do fenômeno. As principais propriedades dos variogramas consistem em: (a) alcance (distância em que as amostras passam a ser independentes, fato que ocorre a partir alcance do patamar.); (b) patamar (valor que designa a estabilização do variograma no campo aleatório.); (c) continuidade na origem; (d) anisotropia (Matheron, 1963).

Por meio da análise variográfica é possível identificar fenômenos anisotrópicos, ou seja, a ocorrência da variação do comportamento do variograma com a direção em que é calculado. Há, desta maneira, direções preferenciais de variabilidade, sendo a direção preferencial aquela que mostra maior continuidade. Possíveis descontinuidades na origem podem ocorrer, apresentando-se o efeito pepita. Este é decorrente de erros de amostragem, de análise ou é proveniente da variabilidade natural do meio amostrado (Matheron, 1963; Yamamoto, 2001). Depois de calcular o variograma experimental, procede-se ao ajuste de uma função matemática representada por um modelo teórico do variograma que descreve continuamente a variabilidade espacial dos dados a partir dos parâmetros e dos pontos do variograma experimental. A partir desta definição, procede-se à escolha de um método para estimativas, como as krigagens.

O método da krigagem tende a suavizar detalhes e valores extremos do conjunto de dados originais (Deutsch \& Journel, 1992). Neste presente estudo, optouse inicialmente pelo uso da krigagem ordinária (Figura 2), técnica que recorre a ponderadores lineares para combinar a informação amostrada, garantindo dois critérios de qualidade: o não-enviesamento, ou seja, a média dos valores estimados num domínio deve ser igual à média dos valores experimentais, e a minimização da variância da estimativa (Isaaks \& Srivastava, 1989; Goovaerts, 1997; Felgueiras et al., 2002). O estimador consiste na média ponderada dos valores, sendo a somatória dos pesos equivalente a 1. A determinação de um valor na posição $x_{0}$ utilizando-se $n$ dados vizinhos $Z\left(x_{i}\right)$ por meio de uma combinação linear com ponderadores $\lambda_{\mathrm{i}}$, é obtida de acordo com a Eq. 1 (Yamamoto, 2001):

$$
\mathrm{z}^{*}\left(\mathrm{x}_{0}\right)=\sum_{\mathrm{i}=1}^{\mathrm{n}} \lambda_{\mathrm{i}}\left(\mathrm{x}_{0}\right) \mathrm{Z}\left(\mathrm{x}_{\mathrm{i}}\right),
$$

onde $Z$ * $\left(x_{0}\right)$ compreende o estimador.

Outra técnica adotada equivaleu à krigagem por indicação (Journel, 1984), caracterizada por ser não paramétrica, ou seja, não considera nenhum tipo de distribuição de probabilidade a priori para a variável aleatória, diferentemente de modelos gaussianos por exemplo (Figura 3). Procedimentos geoestatísticos da krigagem por indicação servem para produzir a previsão de valores sobre uma superfície e, sobretudo, para gerar modelos de incertezas locais para dados geográficos que compartilham uma base de informações (Felgueiras et al., 2002).

A krigagem da indicatriz compreende um tipo não linear da krigagem que utiliza-se da posição de valores dos dados para gerar uma distribuição local em vez de uma distribuição global das propriedades analisadas (Goovaerts, 1997). Esta técnica estima a probabilidade de ocorrência de variáveis discretas. Desta maneira, para os dados contínuos (geoquímica de ouro em solo), os mesmos passaram por uma transformação binária, formulando-se a seguinte variável indicatriz $\left(\mathrm{I}\left(\mathrm{x}_{\mathrm{i}}\right)\right)$ (Eq. 2) (Figura 3):

$$
\begin{aligned}
\mathrm{I}\left(\mathrm{x}_{\mathrm{i}}\right) & =1, \text { se } \mathrm{x}_{\mathrm{i}} \geq 0.50 \mathrm{ppb} \\
& =0, \text { se } \mathrm{x}_{\mathrm{i}}<0.50 \mathrm{ppb}
\end{aligned}
$$

A codificação conduz a um quadro onde os valores assumidos a cada ponto amostral pertença a 0 ou a 1 . Posterior à definição das variáveis indicatrizes realizamse procedimentos similares àqueles utilizados na krigagem ordinária, como a determinação da malha do grid, do variograma experimental das indicatrizes e o ajuste de modelo variográfico. O variograma da indicatriz representa a probabilidade de que, para cada par de pontos separados por uma distância $h$, um deles pertença a 1 ou a 0 , sendo o valor máximo do patamar equivalente a 1.

A etapa seguinte equivale à estimativa de uma superfície 2D, a partir dos dados variográficos das indicatrizes, sendo que valores não amostrados ocorrem no intervalo entre 0 e 1, ou seja, locados entre $0 \%$ e $100 \%$ de probabilidade de pertencerem às maiores anomalias auríferas. A partir do mapa da variável indicatriz krigado é possível determinar cortes de probabilidades para as anomalias de ouro em solo, sedimentos de corrente e em rocha, a fim de comparações de resultados e de áreas mais anômalas para a prospecção, procedimento incluso na análise de incerteza.

A análise de modelos de incerteza foca a inferência das distribuições de probabilidade para cada posição do espaço considerado. O que interessa não é inferir exatamente um certo valor, porém definir áreas com maior probabilidade que o evento ocorra (Felgueiras et al., 2002). Para tal, são determinados os níveis de corte a partir dos quais serão obtidos os mapas de probabilidade de ocorrência; no estudo de caso, os cortes estabelecidos consistem em $10 \%, 25 \%$ e $50 \%$.

\section{Simulação estocástica dos dados geoquímicos}

Como visto anteriormente, a krigagem gera estimativas pontuais, ou seja, independentemente das estimativas vizinhas, compreendendo a melhor estimativa local associada a um variância de erro mínima possível. O mapa, entretanto, pode não ser o melhor como um todo, visto que a técnica tende a suavizar dados, observandose, tipicamente, as seguintes situações: baixos valores amostrais tendem a ser superestimados e os altos valores subestimados. Isto é uma problemática quando o 
objetivo compreende a verificação de padrões de valores extremos, como anomalias auríferas, por exemplo. Além disso, a suavização não é uniforme e é dependente da configuração dos dados. Um mapa krigado também parece mais variável quando a área é mais amostrada, e menos quando há porções pouco amostradas. Isto pode gerar artefatos na imagem krigada final (Goovaerts,

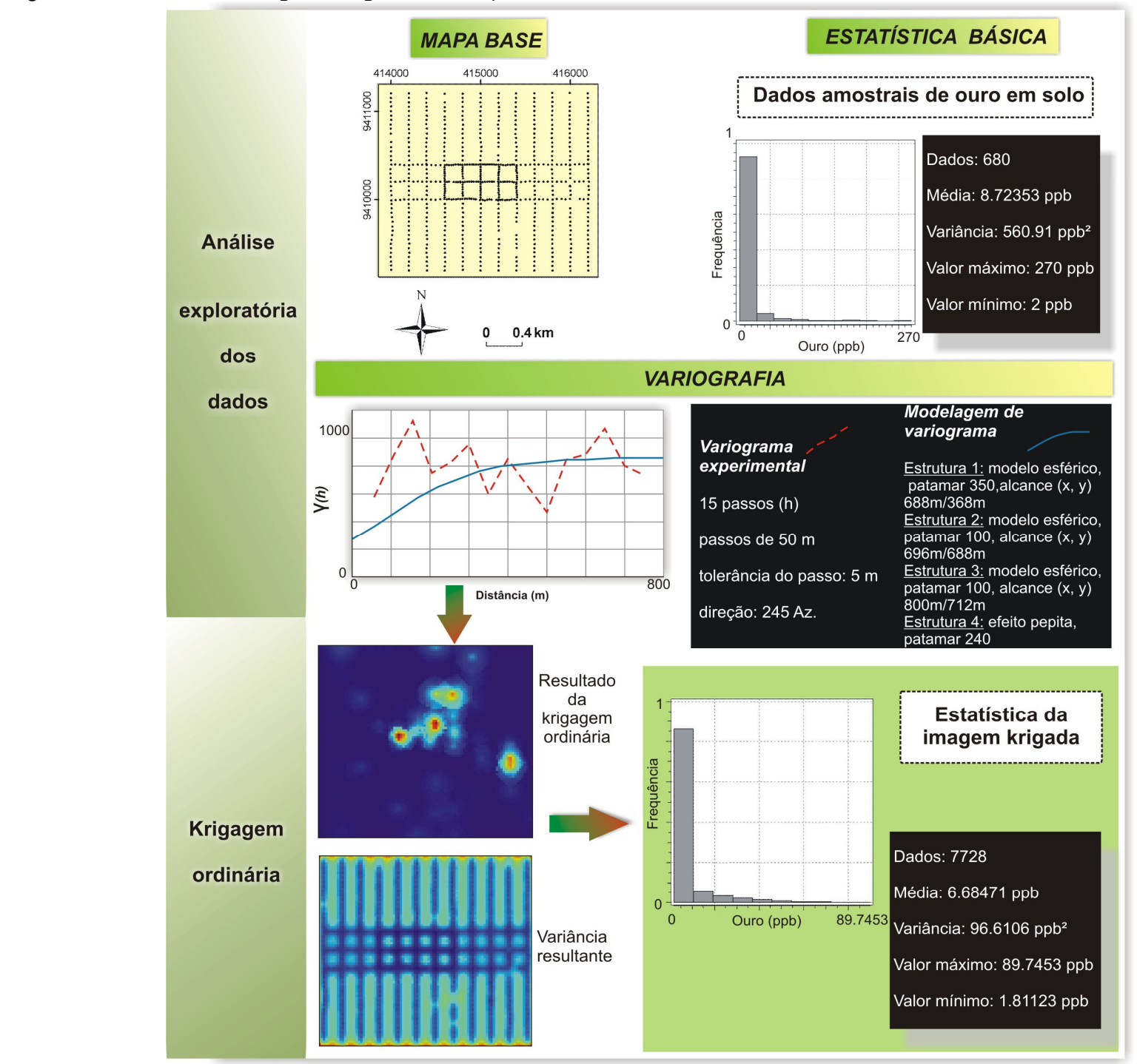

Figura 2. Aplicação da krigagem ordinária aos dados amostrados de ouro em solo.
1997). Por isso, para fins de avaliação de dados extremos, a krigagem não é muito indicada. Objetivandose um produto mais eficiente para a questão, aparecem as simulações estocásticas, que se valem da geração de $\mathrm{L}$ realizações $\mathrm{z}\left({ }^{\prime}\right)(\mathrm{u}), l=1, \ldots, \mathrm{L}$ para células u (Goovaerts, 1997).
As simulações estocásticas foram idealizadas para a reprodução da variabilidade da variável regionalizada em estudo de maneira probabilística. A medida da incerteza espacial dos valores amostrados é feita de forma conjunta e não individual como na krigagem (Goovaerts, 1997). O objetivo da aplicação destas simulações compreende a reprodução da textura geológica (características globais) a partir da produção de modelos ilimitados (L realizações), onde cada um é uma representação da realidade, fato que permite obter a incerteza de forma mais realística acerca das heterogeneidades da variável. Desta maneira, não se produz o cenário mais provável dos valores de uma variável regionalizada, mas sim imagens equiprováveis com mesma média, histograma e variabilidade espacial dos valores experimentais (Goovaerts, 1997).

Dentre os grupos de simulações destacam-se as sequenciais que, em suma, baseiam-se na extensão do condicionamento a toda a informação existente na vizinhança do ponto a simular e que é fornecida tanto pelos valores amostrados da variável quanto pelos valores simulados anteriormente. Esta relação pode ser 
observada pela simulação do primeiro valor $\mathrm{z}_{1}$ a partir da distribuição $\mathrm{F}\left(\mathrm{x}_{1} ; \mathrm{z}_{1} \mid(\mathrm{n})\right)$, seguindo-se da simulação do segundo valor $z_{2}$ por meio da distribuição condicional $F$ $\left(x_{2} ; z_{2} \mid(n+1)\right)$, onde $n+1$ equivale ao condicionamento aos $\mathrm{n}$ dados experimentais acrescidos do valor simulado na simulação anterior. O princípio sequencial é utilizado para simular variáveis categóricas (Simulação Indicatriz Sequencial - SIS) ou contínuas (Simulação Gaussiana Sequencial - SGS)

A SIS compreende a técnica não-gaussiana mais comumente utilizada. A produção da variável indicatriz, do variograma experimental e modelado é realizada da mesma forma quando se aplica a krigagem indicatriz
(Figura 3): este enfoque permite identificar padrões de uma classe específica com continuidade espacial por meio de diferentes modelos de semivariograma (Goovaerts, 1997). A análise dos resultados das $n$ simulações somente é realizada com o pósprocessamento a fim de analisar somente um resultado, a exemplo do cálculo da média, desvio-padrão, além de cortes de probabilidade. No estudo de caso no prospecto 12 de Outubro, optou-se pela produção de 500 imagens simuladas e cálculo da imagem da média destas simulações, além de cortes de probabilidades de $10 \%$, $25 \%$ e $50 \%$ (Figura 4 ).

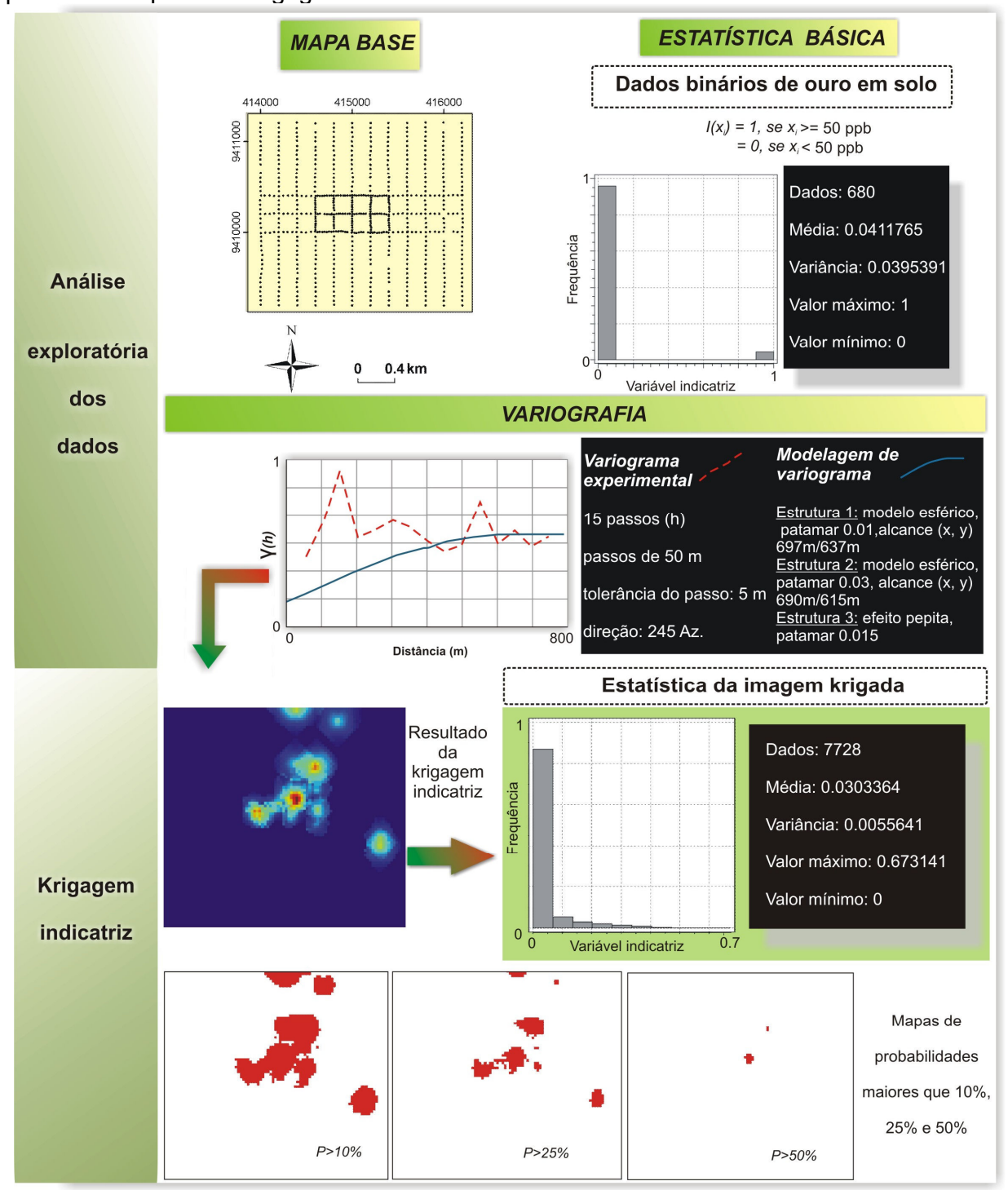

Figura 3. Aplicação da krigagem indicatriz aos dados amostrados de ouro em solo.

\section{Resultados e discussões}

A aplicação das técnicas da krigagem ordinária, indicatriz e da simulação indicatriz seqüencial então apresentadas, respectivamente, nas figuras 2,3 e 4 .
Com relação ao uso das krigagens (Figuras 2 e 3), notase que a estatística das imagens geradas em relação à dos dados são subestimadas, ou seja, valores de 
máximo, mínimo, variância e média das imagens produzidas pelas krigagens ordinária e indicatriz são inferiores aos dos dados amostrais. Este efeito de suavização dos dados é uma característica inerente ao processamento de dados via krigagens, e afeta a compreensão dos valores geoquímicos anômalos em um programa de exploração mineral.

Apesar de ser pouco empregada nas geociências, as simulações estocásticas são interessantes pelo fato principal de evitar a suavização extrema dos dados originais. No presente estudo, 500 imagens foram geradas, todas caracterizadas por estatística básica a mais próxima possível dos dados amostrais sendo, porém, visualmente diferenciadas (Figura 4). Exemplos podem ser vistos na figura 4 a partir de três cenários equiprováveis designados como SIS01, SIS101 e SIS209. O resultado que deve ser interpretado por meio do emprego da simulação indicatriz seqüencial compreende aquele derivado de um pós-processamento, como a média das $n$ imagens simuladas geradas e cortes de probabilidades. Estes dois tipos de resultados foram produzidos, conforme mostrado na figura 4 .

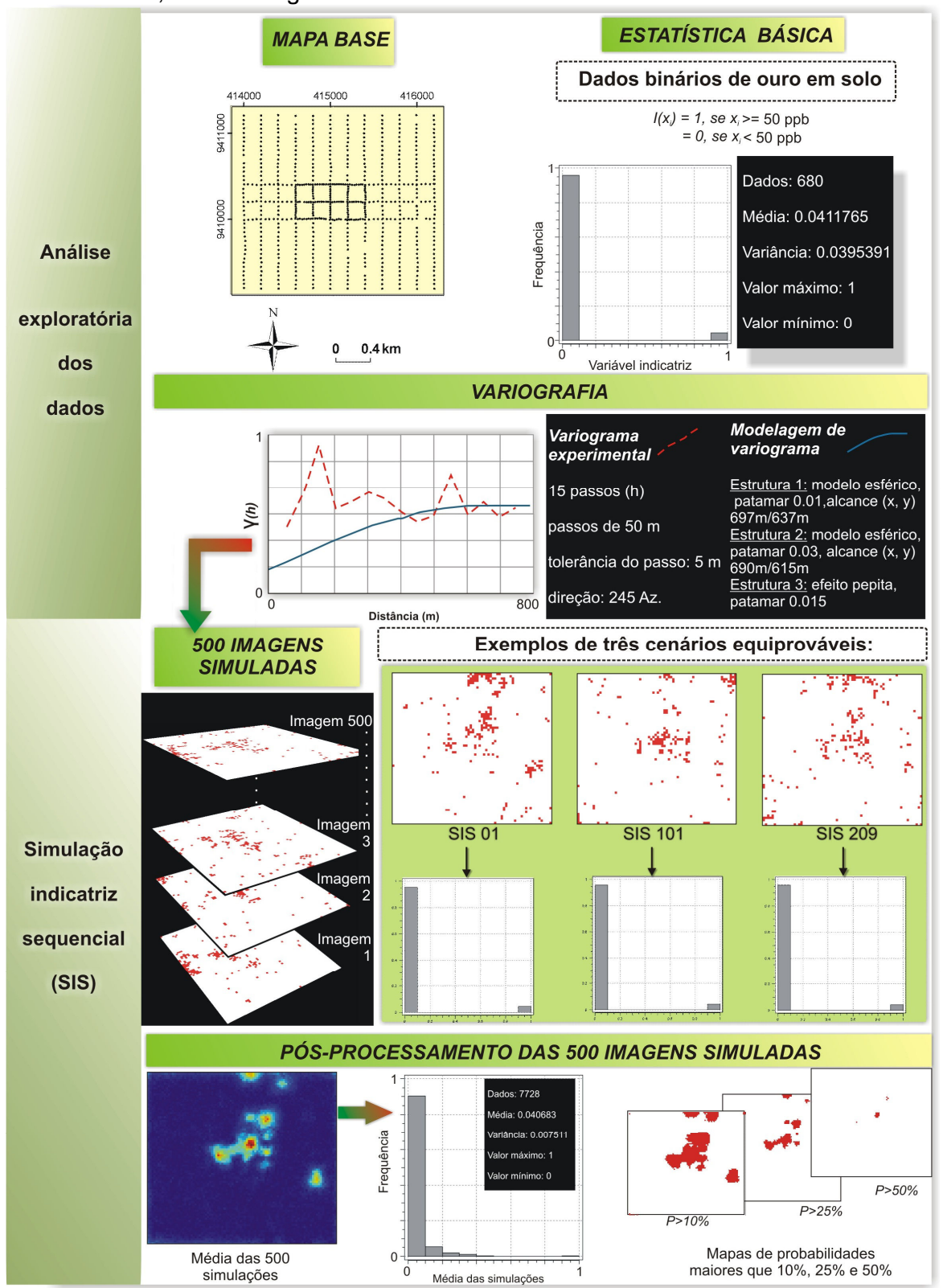

Figura 4. Aplicação da simulação indicatriz sequencial aos dados amostrados de ouro em solo.

Analisando a imagem da média das 500 imagens simuladas (Figuras 4 e 5), nota-se que esta se caracteriza por manter as heterogeneidades dos dados amostrais, ao invés das grandes regiões anômalas de 
ouro com o formato semi-circular típico que as krigagens geram (Figuras 2 e 3). A análise estatística da imagem da média das 500 simulações evidencia uma diminuição da variância $(0,0075)$ e a manutenção do valor mais anômalo como sendo 1 , honrando os dados amostrais. Desta forma, a imagem da média das simulações indica a variação entre $0 \%$ e $100 \%$ da probabilidade de ocorrência de anomalias de ouro maiores que $50 \mathrm{ppb}$. Cortes de probabilidades maiores que $10 \%, 25 \%$ e $50 \%$ também foram realizados a partir da imagem da média das 500 simulações, objetivando a averiguação de áreas mais anômalas (Figura 4).

Destaca-se, entretanto, que a geração de imagens via simulação estocástica necessita de uma demanda computacional maior, e assim, maior tempo de processamento dos dados.

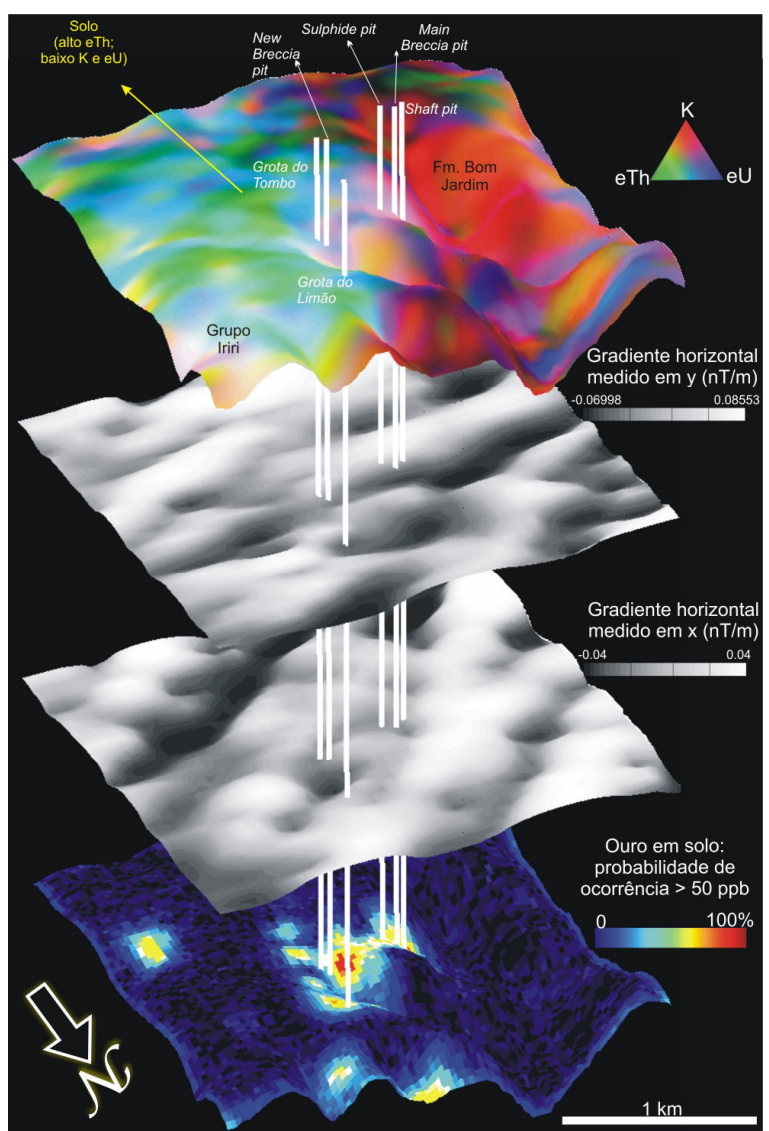

Figura 5. Integração de imagens gamaespectrométrica e dos gradientes horizontais medidos na direção x e y com a imagem de ouro em solo gerada pela simulação indicatriz sequencial, e os pits do prospecto 12 de Outubro.

\section{Conclusões}

Neste trabalho fica evidente que o uso de simulação estocástica, exemplificada pelo emprego da SIS, compreende uma poderosa ferramenta ainda pouco empregada na geração de mapas geoquímicos de interesse à prospecção mineral. Com base nesta técnica, é possível preservar os valores geoquímicos amostrais, o que não é possível em relação a outros métodos suavizadores, como as krigagens, potencializando a integração destes dados com os de geofísica (e.g., Figura 5), para determinação do corpo de minério. Conforme indicado na Figura 5, a integração de imagens gamaespectrométricas e magnetométricas gradiométricas com os pits e a imagem de ouro em solo produzida pela média das 500 simulações, permite averiguar que as ocorrências auríferas atualmente constatadas estão associadas a baixos gradientes magnéticos de direção ENE-WSW e a valores médios a altos de $\mathrm{K}$, eTh e eU associados aos riolitos do Grupo Iriri $(\sim 1,8 \mathrm{Ga})$, hospedeiros das mineralizações.

\section{Agradecimentos}

Agradecemos à UnB pelo suporte técnico, ao CNPq pelo projeto CT-Mineral ( $n^{\circ}$. 555063/2006-2), e à CAPES pela bolsa de mestrado. A.M. Silva e N.F.Botelho agradecem ao $\mathrm{CNPq}$ pelas respectivas bolsas de produtividade em pesquisa.

\section{Referências}

Deutsch C.V. \& Journel A.G. 1992. GSLIB Geostatistical software library and user's guide. Oxford University Press, Nova York, 340 pp.

Felgueiras C.A, Druck S., Monteiro A.M.V. 2002. Análise espacial de superfícies: o enfoque da geoestatística por indicação. Ministério da Ciência e Tecnologia/INPE, 25 p.

Goovaerts P. 1997. Geostatistics for natural resources evaluation. London, Oxford University Press, 483 p.

Isaaks E.H., Srivastava R.M. 1989. Ordinary kriging. In: Isaaks E.H., Srivastava R.M. (ed). An introduction to applied geostatistics. Nova York, Oxford University Press, 278-322.

Joumel, A.G. 1984. The Place of Non-parametric geostatistics. In: G. Verly et al. (Ed.). Geostatistics for Natural Resources Characterization, Part I. D. Reidel Publishing Co., Dordrecht, 307-335.

Klein E.L., Almeida M.E., Vasquez M.L., Bahia R.B.C., Santos M.L.E., Ferreira A.L. 2001a. Geologia e recursos minerais da Província Mineral do Tapajós. Folhas Vila Mamães Anã (SB.21-V-D), Jacareacanga (SB.21-Y-B), Caracol (SB.21-X-X), Vila Riozinho (SB.21-Z-A) e Rio Novo (SB.21-Z-C). Estados do Pará e Amazonas. Escala 1:500.000. Brasília: CPRM/DIEDIG/DEPAT, 2001, 81 p.

Matheron G. 1963. Principles of geostatistics. Economic Geology, vol. 58: 1276-1266.

Yamamoto J.K. 2001. Análise geoestatística. In: Yamamoto J.K. (ed.) Avaliação e classificação de reservas minerais. São Paulo-SP, Ed. Universidade de São Paulo, 69-92. 